\title{
Generating functions for the area below some lattice paths
}

\author{
Donatella Merlini \\ Dipartimento di Sistemi e Informatica, Via Lombroso 6/17, 50134, Firenze, Italia \\ merliniedsi.unifi. it
}

\begin{abstract}
We study some lattice paths related to the concept of generating trees. When the matrix associated to this kind of trees is a Riordan array $D=(d(t), h(t))$, we are able to find the generating function for the total area below these paths expressed in terms of the functions $d(t)$ and $h(t)$.
\end{abstract}

Keywords: Generating functions, Riordan arrays, lattice paths, generating trees, area, internal path length.

\section{Introduction}

In this paper we consider a model of random walks previously studied in [BM02]: each walk starts (at time 0 ) from a point $p_{0}$ of $\mathbb{Z}$ and at time $n$, one makes a jump $x_{n} \in \mathbb{Z}$; the new position is given by the recurrence $p_{n}=p_{n-1}+x_{n}$ where, when $p_{n-1}=k$, the $x_{n}$ 's are constrained to belong to a fixed set $\mathcal{P}_{k}$ (that is, the possible jumps depend on the position of the walk). We call paths on $\mathbb{Z}$ the walks under this model.

In combinatorics, it is classical to represent a particular walk as a path in a two dimensional lattice, thus the drawing corresponds to the walk (of length $n)$ linking the points $\left(\left(0, p_{0}\right),\left(1, p_{1}\right), \ldots,\left(n, p_{n}\right)\right)$. It is also convenient to represent all the walks of length $\leq n$ as a tree of height $n$, where the root (at level 0 by convention) is labeled with the starting point of the walks and where the label of each node at level $n$ encodes a possible position of the walk. Figure 1 illustrates the generating tree representing the walks on $\mathbb{N}$ with jumps $\mathcal{P}=\{+1,-1\}$ starting in 0 (and up to length $n=4)$. The branches $(0,1,0,1,0),(0,1,0,1,2)$, $(0,1,2,1,0),(0,1,2,1,2),(0,1,2,3,2)(0,1,2,3,4)$ correspond to the well-known Dyck paths of length 4 , and are drawn in Figure 2.

This kind of trees are known in the literature as generating trees and in the last years have been widely studied. They have been used for the first time, without any specific name, in [CGHK78] and successively this concept can be found in [Wes95, Wes96]. Generating trees are a device to represent the development of many classes of combinatorial objects which can then be enumerated by counting the different labels in the various levels of the tree (see, e.g., $\left[\mathrm{BBMD}^{+} 02\right.$, BLPP99]).

These walks on $\mathbb{Z}$ are homogeneous in time, since the set of jumps when one is at altitude $k$ is independent from the time. When the positions $p_{n}$ 's are constrained to be nonnegative, we talk about paths on $\mathbb{N}$ (this corresponds to deal with generating trees with positive labels).

When the sets $\mathcal{P}_{k}$ 's are equal to a fixed set $\mathcal{P}$, the corresponding walks have been deeply studied both in combinatorics and in probability theory (see, e.g., [BF02] and the included references); in particular, these walks can be generated by context-free grammars (see, e,g, [MRSV99] ). 


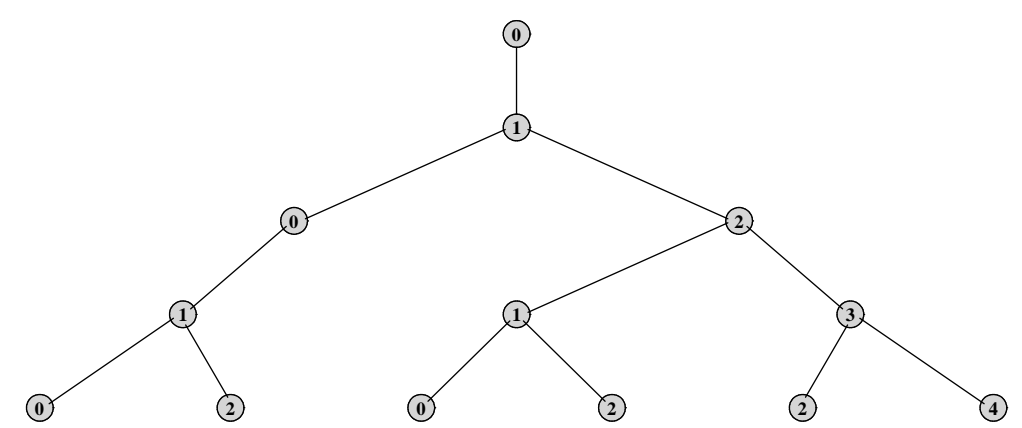

Fig. 1: The generating tree of the walk on $\mathbb{N}$ with jumps $\mathcal{P}=\{+1,-1\}$ starting in 0 (and up to length $n=4$ ). Each branch corresponds to a path.
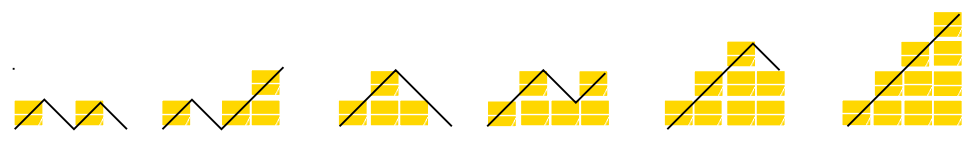

Fig. 2: Dyck paths of length 4 and their area.

When the sets $\mathcal{P}_{k}$ 's are unbounded, walks are not homogeneous in space, since the set of available jumps depends on the position, and it is not possible to generate them by context-free grammars. However, if the sets $\mathcal{P}_{k}$ 's have a "combinatorial" shape, it is reasonable to hope that the generating function associated to the corresponding walk would have some nice properties. In [BM02], several classes of such walks are presented and the nature of the generating function counting the number of walks of length $n$ going from 0 to $k$ (or, equivalently, the number of nodes with label $k$ at level $n$ in the tree) is studied.

In this paper, we examine the walks on $\mathbb{N}$ related to the concept of proper Riordan arrays and study, in particular, the area below these paths and the $x$-axis.

The concept of Riordan arrays provides a remarkable characterization of many lower triangular arrays that arise in combinatorics. The theory has been introduced in [SGWW91] and then examined closely from a theoretical and practical viewpoint in [Spr94, MRSV97]. Recently, in [MV00], the connection between proper Riordan arrays and generating trees has been investigated and the resulting trees are called proper generating trees; this relation allows to combine the counting capabilities of both approaches and can be exported in the context of lattice paths.

The area below paths is a combinatorial problem which has some important connections with permutations and the internal path length in various types of trees and has been studied in several contexts (see, e.g., [BK01, DF93, GJ83, Knu73, MSV96, Sul98, Sul00]).

As it will be shown in Section 2, the total area below all paths on $\mathbb{N}$ of length $n$ is related to the total internal path length, weighted with the values of the labels in the nodes and up to level $n$, of the corresponding generating tree. The internal path length of proper generating trees has been studied in [Mer02]; here, we present similar results in the context of lattice paths thus finding an explicit generating function for the total area below all the paths under the present model and, in particular, for those with an infinite set of jumps. The involved generating functions are expressed in terms of the functions $(d(t), h(t))$ defining the associated proper Riordan array (see Theorem 4). 


\section{Background}

In this section we summarize some results on generating trees and Riordan arrays which will be useful in the next sections. The complete theory of Riordan arrays, the proofs of their properties and the relation with generating trees can be found in [MRSV97, MV00].

\subsection{Generating trees}

A generating tree is a rooted labeled tree with the property that if $v_{1}$ and $v_{2}$ are any two nodes with the same label then, for each label $l, v_{1}$ and $v_{2}$ have exactly the same number of children with label $l$. In order to specify a generating tree we have to specify a label for the root and a set of rules explaining how to derive from the label of a parent the labels of all of its children. For example, Figure 1 illustrates the upper part of the generating tree which corresponds to the following specification:

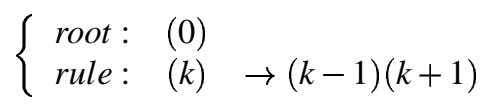

We can associate a matrix to any generating tree: a matrix associated to a generating tree (AGT matrix, for short) is an infinite matrix $\left\{d_{n, k}\right\}_{n, k \in \mathbb{N}}$ where $d_{n, k}$ is the number of nodes at level $n$ with label $k+c, c$ being the label of the root.

For example, for rule (1) we have the following AGT matrix:

\begin{tabular}{c|ccccc}
$n / k$ & 0 & 1 & 2 & 3 & 4 \\
\hline 0 & 1 & & & & \\
1 & 0 & 1 & & & \\
2 & 1 & 0 & 1 & & \\
3 & 0 & 2 & 0 & 1 & \\
4 & 2 & 0 & 3 & 0 & 1
\end{tabular}

In our model of random walks, assuming $c=0$ (this choice will be explained later), the quantity $d_{n, k}$ in the AGT matrix represents the number of paths of length $n$, starting at the origin and ending at altitude $k$.

Many AGT matrices can be studied from a Riordan array point of view.

\subsection{Riordan arrays}

A Riordan array is an infinite lower triangular array $\left\{d_{n, k}\right\}_{n, k \in \mathbb{N}}$, defined by a pair of formal power series $D=(d(t), h(t))$, such that the generic element $d_{n, k}$ is the $n$-th coefficient in the series $d(t)(t h(t))^{k}$, i.e.:

$$
d_{n, k}=\left[t^{n}\right] d(t)(\operatorname{th}(t))^{k}, \quad n, k \geq 0 .
$$

From this definition we have $d_{n, k}=0$ for $k>n$. The bivariate generating function of a Riordan array is given by:

$$
d(t, w)=\sum_{n, k \geq 0} d_{n, k} t^{n} w^{k}=\frac{d(t)}{1-w t h(t)} .
$$

In the sequel we always assume that $d(0) \neq 0$; if we also have $h(0) \neq 0$ then the Riordan array is said to be proper; in the proper-case the diagonal elements $d_{n, n}$ are different from zero for all $n \in \mathbb{N}$. The most 
simple example is the Pascal triangle for which we have

$$
\left(\begin{array}{l}
n \\
k
\end{array}\right)=\left[t^{n}\right] \frac{1}{1-t}\left(\frac{t}{1-t}\right)^{k}
$$

where we recognize the proper Riordan array $d(t)=h(t)=1 /(1-t)$ or $d(t, w)=1 /(1-t(1+w))$, as can be easily proved from (2) and (3).

Proper Riordan arrays can also be defined in terms of two sequences $A=\left\{a_{i}\right\}_{i \in \mathbb{N}}$ with $a_{0} \neq 0$ and $Z=\left\{z_{0}, z_{1}, z_{2}, \ldots\right\}$ (see, [Rog78, Spr94, MRSV97]) such that every element $d_{n+1, k+1}$ can be expressed as a linear combination, with coefficients in $A$, of the elements in the preceding row, starting from the preceding column:

$$
d_{n+1, k+1}=a_{0} d_{n, k}+a_{1} d_{n, k+1}+a_{2} d_{n, k+2}+\cdots
$$

and such that every element in column 0 can be expressed as a linear combination, with coefficients in $Z$, of all the elements of the preceding row:

$$
d_{n+1,0}=z_{0} d_{n, 0}+z_{1} d_{n, 1}+z_{2} d_{n, 2}+\cdots .
$$

The generating functions $A(t)$ and $Z(t)$ of these sequences are related to the pair $(d(t), h(t))$ by the following formulas:

$$
\begin{gathered}
h(t)=A(\operatorname{th}(t)) \\
d(t)=\frac{d_{0}}{1-t Z(t h(t))}
\end{gathered}
$$

For example, for the Pascal triangle we have: $A(t)=1+t$ and $Z(t)=1$.

Another interesting result concerns the computation of combinatorial sums involving Riordan arrays:

Theorem 1. Let $D=(d(t), h(t))$ be a Riordan array and $f(t)$ the generating function for the sequence $\left\{f_{k}\right\}_{k \in \mathbb{N}}$. Then:

$$
\sum_{k=0}^{n} d_{n, k} f_{k}=\left[t^{n}\right] d(t) f(t h(t))
$$

In particular, when $f_{k}=1$ we have:

$$
\sum_{k=0}^{n} d_{n, k}=\left[t^{n}\right] \frac{d(t)}{1-\operatorname{th}(t)}
$$

and this formula can be used, in the present context, to compute the total number of paths of length $n$. In fact, the following connection between proper Riordan array and generating trees holds:

Theorem 2. Let $c \in \mathbb{N}, a_{j}, z_{j} \in \mathbb{N}, \forall j \geq 0, a_{0} \neq 0$ and $k \geq c$ and let

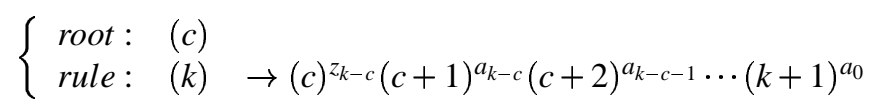

be a generating tree specification. Then, the AGT matrix associated to (7) is a proper Riordan array $D$ defined by the triple $\left(d_{0}, A, Z\right)$, such that

$$
d_{0}=1, \quad A=\left(a_{0}, a_{1}, a_{2}, \ldots\right), \quad Z=\left(z_{0}, z_{1}, z_{2}, \ldots\right) .
$$


On the contrary, if $D$ is a proper Riordan array defined by the triple $\left(d_{0}, A, Z\right)$ with $d_{0}=1$ and $a_{j}, z_{j} \in \mathbb{N}$, $\forall j \geq 0$, then $D$ is the AGT matrix associated to the generating tree specification (7).

Note we only consider nonnegative labels, thus when a rule gives a negative value, we simply ignore this label. Moreover, the powers in the rule denote repetition of the same label, so we write $(k)^{r}$ instead of $\underbrace{(k)(k) \ldots(k)}_{r}$.

As an application of the previous theorem, let us consider the rule (1), the first few applications of which give:

$$
(0) \rightarrow(1), \quad(1) \rightarrow(0)(2), \quad(2) \rightarrow(1)(3), \quad \cdots
$$

We thus recognize rule (7) with $A=(1,0,1,0,0, \ldots)$ and $Z=(0,1,0,0,0 \ldots)$, that is, $A(t)=1+t^{2}$ and $Z(t)=t$. By applying formulas (4) and (5) we find that the pair $(d(t), h(t))$ defining the AGT matrix for the rule (1) corresponds to:

$$
d(t)=h(t)=\frac{1-\sqrt{1-4 t^{2}}}{2 t^{2}} .
$$

Formula (6) in this case gives the following generating function:

$$
\frac{1-2 t-\sqrt{1-4 t}}{2 t(2 t-1)}=1+t+2 t^{2}+3 t^{3}+6 t^{4}+10 t^{5}+20 t^{6}+35 t^{7}+70 t^{8}+O\left(t^{9}\right)
$$

\section{The area below proper paths on $\mathbb{N}$}

In this section we examine paths on $\mathbb{N}$ described by the rule (7) with $c=0$. The root of a generating trees can have any label and in fact in [Mer02] the internal path length has been studied for a generic label $c$ in the root. In the present context, since the label of the root represents the starting point of each path, we can always assume $c=0$ : different values of this label correspond to translate each path, along the $y$-axis, by the same quantity. From here on, we will call proper paths on $\mathbb{N}$ the paths described by the following rule:

$$
\left\{\begin{array}{ll}
\text { root }: & (0) \\
\text { rule }: & (k)
\end{array} \rightarrow(0)^{z_{k}}(1)^{a_{k}}(2)^{a_{k-1}} \cdots(k+1)^{a_{0}}\right.
$$

where, according to Theorem $2, A=\left(a_{0}, a_{1}, a_{2}, \ldots\right)$ and $Z=\left(z_{0}, z_{1}, z_{2}, \ldots\right)$ are the $A$ and $Z$-sequences of the associated AGT matrix.

We explicitly observe that when the generating functions $A(t)$ and $Z(t)$ are polynomials, that is, $A$ and $Z$ have a finite number of coefficients different from zero, then the generating tree corresponding to rule (8) defines walks on $\mathbb{N}$ with a finite set of jumps. More generally, (8) defines walks with an infinite set of jumps, which depends on the position. The powers in the rule can be interpreted as colors that can be used to distinguish various occurrences of the same jump.

Note, in particular, the jump +1 is the only positive jump allowed and it always belongs to the set of available jumps since, by hypothesis, $a_{0} \neq 0$.

Our interest consists in computing the total area between the paths of length $n$ and the x-axis; this quantity is related to the total internal path length, up to level $n$, in the corresponding generating tree, weighted with the value of each node label. Referring to Figure 1, we have a total path length equal to 1 for paths up to level 1, equal to 4 for paths up to level 2, equal to 12 for paths up to level 3 and equal to 
34 for paths up to level 4 . In fact, we will prove that the generating function counting the total path length for rule (1) is given by:

$$
P(t)=\frac{1+t-\sqrt{1-4 t^{2}}}{(1-2 t)\left(1-4 t^{2}\right)}=t+4 t^{2}+12 t^{3}+34 t^{4}+84 t^{5}+212 t^{6}+488 t^{7}+1162 t^{8}+O\left(t^{9}\right) .
$$

Any path of length $n$ in a proper generating tree can be seen as a histogram of length $n$ : in fact any label $k$ in the path can be associated to a column of $k$ cells and by juxtaposing several columns in such a way that their lowest cells are at the same level, we obtain what we call a histogram. Thus, the total internal path length corresponds to the total area of histograms, if we compute the area as the sum of the columns height. On the other hand, it is evident that the area of these histograms is strictly related to the area of the regions between the paths and the $x$-axis, as shown for example in Figure 2 for Dyck paths of length 4. We therefore define the area $A(W)$ of a path

$$
W=\left(\left(0, p_{0}\right),\left(1, p_{1}\right), \ldots,\left(n, p_{n}\right)\right)
$$

on $\mathbb{N}$ as the sum of the ordinates of its points:

$$
A(W)=\sum_{i=0}^{n} p_{i}
$$

In this paper, we are interested in the generating function $P(t)=\sum_{n \geq 0} P_{n} t^{n}$ counting the total area $P_{n}$ of all the paths of length $n$ described by rule (8). In particular, we'll find a formula which only depends on the functions $d(t)$ and $h(t)$ defining the associated proper Riordan array. More generally, one can study the $j^{\text {th }}$ moment $A(W, j)=\sum_{i=0}^{n} p_{i}^{j}$ of a path $W$. The method we propose in this section can be used to find the total moments of any order $j$ for all the paths of length $n$ but the computations in this case become very complicated.

In order to study the total area of proper paths on $\mathbb{N}$, we consider the total internal path length of the corresponding generating tree. The total sum of the labels in all the paths from level 0 to level $n$ in the generating tree can be computed, level by level, by summing the labels counted with their multiplicity; if $P_{i, n}$ is the sum of the labels at level $i$ counted with their multiplicity we have:

$$
P_{n}=\sum_{i=0}^{n} P_{i, n}
$$

Figure 3 illustrates how $P_{i, n}$ can be computed: if we fix level $n$ and consider a label $(r)$ at level $i, 0 \leq i \leq n$, the multiplicity of this label is given by the number of nodes at level $n-i$ in the marked sub-tree, that is, in the generating tree having the same specification (7) but root labeled $(r)$. This quantity must be multiplied by the number of nodes at level $i$ having label $(r)$ and obviously by the value $r$ of the label. On the other hand, we know that the element $d_{i, r}$ of the associated proper Riordan array counts the number of nodes at level $i$ with label $(r)$. So, if we let $f_{j}(t)$ be the generating function counting the number of nodes at a given level in the generating tree having root labeled $(j)$ we have:

$$
P_{n}=\sum_{i=0}^{n} P_{i, n}=\sum_{i=0}^{n} \sum_{r \geq 0} d_{i, r} r\left[t^{n-i}\right] f_{r}(t) .
$$

The first step in the computation of the sum (9) consists in the computation of the generating function $f_{j}(t)$. 


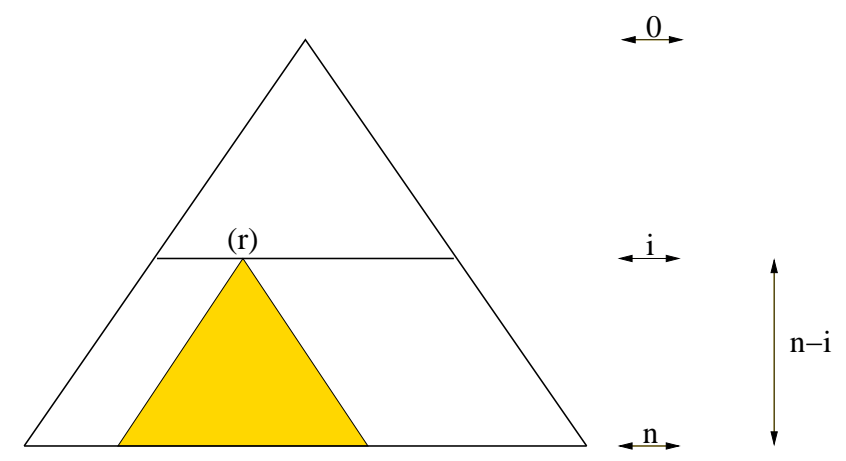

Fig. 3: The computation of the area.

Theorem 3. Let $f_{j}(t)$ be the generating function counting the number of nodes at a given level in the generating tree (8) having root labeled $(j)$. We have:

$$
f_{0}(t)=\frac{d(t)}{1-t h(t)}, \quad f_{r}(t)=\frac{p_{r}(t) f_{0}(t)-q_{r}(t)}{a_{0}^{r} t^{r}}
$$

where

$$
\begin{gathered}
p_{0}(t)=1, \quad p_{r}(t)=\sum_{k=0}^{r} p_{r, r-k} t^{k} \\
q_{0}(t)=0, \quad q_{r}(t)=\sum_{k=0}^{r-1} q_{r-1, r-1-k} t^{k} .
\end{gathered}
$$

Moreover, the matrices $P=\left\{p_{r, k}\right\}_{r, k \in \mathbb{N}}$ and $Q=\left\{q_{r, k}\right\}_{r, k \in \mathbb{N}}$ correspond to the following proper Riordan arrays:

$$
\begin{gathered}
P=\left(d_{P}(t), h_{P}(t)\right)=\left(1-\frac{a_{0} t Z\left(a_{0} t\right)}{A\left(a_{0} t\right)}, \frac{a_{0}}{A\left(a_{0} t\right)}\right), \\
Q=\left(d_{Q}(t), h_{Q}(t)\right)=\left(\frac{a_{0}}{\left(1-a_{0} t\right) A\left(a_{0} t\right)}, \frac{a_{0}}{A\left(a_{0} t\right)}\right) .
\end{gathered}
$$

Proof. See Theorems 3.2 and 3.3 in [Mer02]

The Riordan array nature of $f_{j}(t)$ is useful to compute the bivariate generating function $F(t, w)=$ $\sum_{r \geq 0} f_{r}(t) w^{r}$.

In fact, by using Theorems 1 and 3 we have:

$$
\begin{gathered}
F(t, w)=f_{0}(t) \sum_{r \geq 0} p_{r}(t)\left(\frac{w}{a_{0} t}\right)^{r}-\sum_{r \geq 0} q_{r}(t)\left(\frac{w}{a_{0} t}\right)^{r}= \\
=f_{0}(t) P\left(\frac{w}{a_{0}}, \frac{1}{t}\right)-\frac{w}{a_{0} t} Q\left(\frac{w}{a_{0}}, \frac{1}{t}\right)=\frac{t d(t)(1-w)(A(w)-w Z(w))+t w h(t)-w}{(t A(w)-w)(1-t h(t))(1-w)},
\end{gathered}
$$


being $P(t, w)$ and $Q(t, w)$ the bivariate generating functions of the Riordan arrays $P$ and $Q$ defined in Theorem 3 (see formula 3). Now, if we let $G(t, w)=\sum_{r \geq 0} r f_{r}(t) w^{r}$ we simply have

$$
G(t, w)=w \frac{\partial}{\partial w} F(t, w)
$$

hence, by using Theorem 1, we obtain:

$$
\sum_{r \geq 0} d_{i, r} r f_{r}(t)=\sum_{r \geq 0} d_{i, r}\left[w^{r}\right] G(t, w)=\left[w^{i}\right] d(w) G(t, w h(w))
$$

and

$$
P_{n}=\sum_{i=0}^{n} P_{i, n}=\sum_{i=0}^{n}\left[t^{n-i}\right]\left[w^{i}\right] d(w) G(t, w h(w)) .
$$

The generating function $P(t)=\sum_{n \geq 0} P_{n} t^{n}$ can now be computed by putting $t=w$ in $d(w) G(t, w h(w))$. This last computation is made complicated by the presence of a factor $(t-w)^{2}$ at the denominator, but after some computation one finally has the following:

Theorem 4. Let $P(t)=\sum_{n \geq 0} P_{n} t^{n}$ be the generating function counting the total area of the paths defined by the specification rule (8). Then we have:

$$
P(t)=\frac{N_{P}(t)}{D_{P}(t)}
$$

where

$$
\begin{gathered}
N_{P}(t)=\frac{1}{2} t^{2} d(t) h(t) d^{\prime \prime}(t)(1-t h(t))^{2}+\frac{1}{2} t^{3} d(t)^{2} h(t) h^{\prime \prime}(t)(1-t h(t))+ \\
+t^{4} d(t)^{2} h(t) h^{\prime}(t)^{2}-t^{2} h(t) d^{\prime}(t)^{2}(1-t h(t))^{2}+t d(t)^{2} h(t)^{2}+2 t^{2} d(t)^{2} h(t) h^{\prime}(t), \\
D_{P}(t)=d(t)\left(h(t)+t h^{\prime}(t)\right)(1-t h(t))^{3} .
\end{gathered}
$$

\section{Some examples}

In this section we take into consideration some examples of proper paths on $\mathbb{N}$ and find the generating function $P(t)=\sum_{n \geq 0} P_{n} t^{n}$ for their total area. We explicitly observe that by dividing $P_{n}$ by the total number of paths of length $n$ (see formula 6) one can obtain the average area of a path of length $n$ (the probabilistic model under consideration is the uniform distribution on all paths of length $n$ ). We first examine some paths on $\mathbb{N}$ with a finite set of jumps and then conclude with some other examples with an infinite set of jumps.

\subsection{The finite set of jumps $\mathcal{P}=\{-1,0,+1\}$ with colours}

The rule under consideration in this case is $(\alpha \neq 0$ :)

$$
\begin{cases}\text { root : } & (0) \\ \text { rule }: & (k) \rightarrow(k-1)^{\gamma}(k)^{\beta}(k+1)^{\alpha}\end{cases}
$$


This scheme is well-known (see, e.g. [MSV94]) and by choosing $\alpha, \beta, \gamma$ appropriately we recognize famous paths: for example, $(\alpha, \beta, \gamma)=(1,0,1)$ corresponds to Dyck paths and $(\alpha, \beta, \gamma)=(1,1,1)$ corresponds to Motzkin paths. The Riordan array associated to this rule is defined by $A(t)=\alpha+\beta t+\gamma t^{2}$ and $Z(t)=\beta+\gamma t$, hence, for $\gamma \neq 0$, we have:

$$
d(t)=\frac{1-\beta t-\sqrt{1-2 \beta t+\left(\beta^{2}-4 \alpha \gamma\right) t^{2}}}{2 t^{2} \alpha \gamma}, \quad h(t)=\frac{1-\beta t-\sqrt{1-2 \beta t+\left(\beta^{2}-4 \alpha \gamma\right) t^{2}}}{2 t^{2} \alpha}
$$

For $\gamma=0$, instead, we have:

$$
d(t)=\frac{1}{1-\beta t}, \quad h(t)=\frac{\alpha}{1-\beta t} .
$$

The generating function for the total area in terms of generic values of $\alpha, \beta, \gamma$ is quite complex and we prefer to give some special cases in the following Table:

\begin{tabular}{|c|c|}
\hline$(\alpha, \beta, \gamma)$ & $P(t)$ \\
\hline$(1,1,1)$ & $\frac{1-\sqrt{1-2 t-3 t^{2}}}{(1-3 t)^{2}(t+1)}=t+7 t^{2}+34 t^{3}+144 t^{4}+563 t^{5}+O\left(t^{6}\right)$ \\
\hline$(1,2,1)$ & $\frac{1-t-\sqrt{1-4 t}}{(1-4 t)^{2}}=t+10 t^{2}+68 t^{3}+394 t^{4}+2092 t^{5}+O\left(t^{6}\right)$ \\
\hline$(1, \beta, 1)$ & $\frac{1-(\beta-1) t-\sqrt{1-2 \beta t+\left(\beta^{2}-4\right) t^{2}}}{((2+\beta) t-1)^{2}(1-(\beta-2) t)}=t+(4+3 \beta) t^{2}+\left(12+16 \beta+6 \beta^{2}\right) t^{3}+O\left(t^{4}\right)$ \\
\hline$(2,2,1)$ & $\frac{(1-4 t)\left(16 t^{2}-13 t+2\right)-\left(74 t^{2}-25 t+2\right) \sqrt{1-4 t-4 t^{2}}}{4\left(1-4 t-4 t^{2}\right)(1-5 t)^{3}}=2 t+26 t^{2}+232 t^{3}+1768 t^{4}+O\left(t^{5}\right)$ \\
\hline$(\alpha, \beta, 0)$ & $\frac{t \alpha}{(1-(\alpha+\beta) t)^{3}}=\alpha t+3 \alpha(\alpha+\beta) t^{2}+6 \alpha(\alpha+\beta)^{2} t^{3}+10 \alpha(\alpha+\beta)^{3} t^{4}+O\left(t^{5}\right)$ \\
\hline
\end{tabular}

Tab. 1: Functions $P(t)$ corresponding to different $\alpha, \beta, \gamma$ in rule (10).

\subsection{Some infinite sets of jumps}

As a first example of an infinite set of jumps we consider the rule:

$$
\left\{\begin{array}{ll}
\text { root }: & (0) \\
\text { rule }: & (k)
\end{array} \rightarrow(0)^{k}(1)(2) \ldots(k)(k+1)\right.
$$

This rule corresponds to the Riordan array defined by $A(t)=1 /(1-t)$ and $Z(t)=t /(1-t)^{2}$, or, equivalently by

$$
d(t)=\frac{1-5 t+(1-t) \sqrt{1-4 t}}{2\left(1-4 t-t^{2}\right)}, \quad h(t)=\frac{1-\sqrt{1-4 t}}{2 t}
$$

Theorem 4 gives the following generating function for the total area:

$P(t)=\frac{\left(\left(1-12 t+50 t^{2}-86 t^{3}+61 t^{4}-14 t^{5}\right) \sqrt{1-4 t}+1-14 t+72 t^{2}-164 t^{3}+163 t^{4}-78 t^{5}+4 t^{6}\right)(1-\sqrt{1-4 t})}{4(1-4 t)(1+\sqrt{1-4 t})^{3}(1-5 t-(t-1) \sqrt{1-4 t})\left(1-4 t-t^{2}\right)^{2}}=$ 


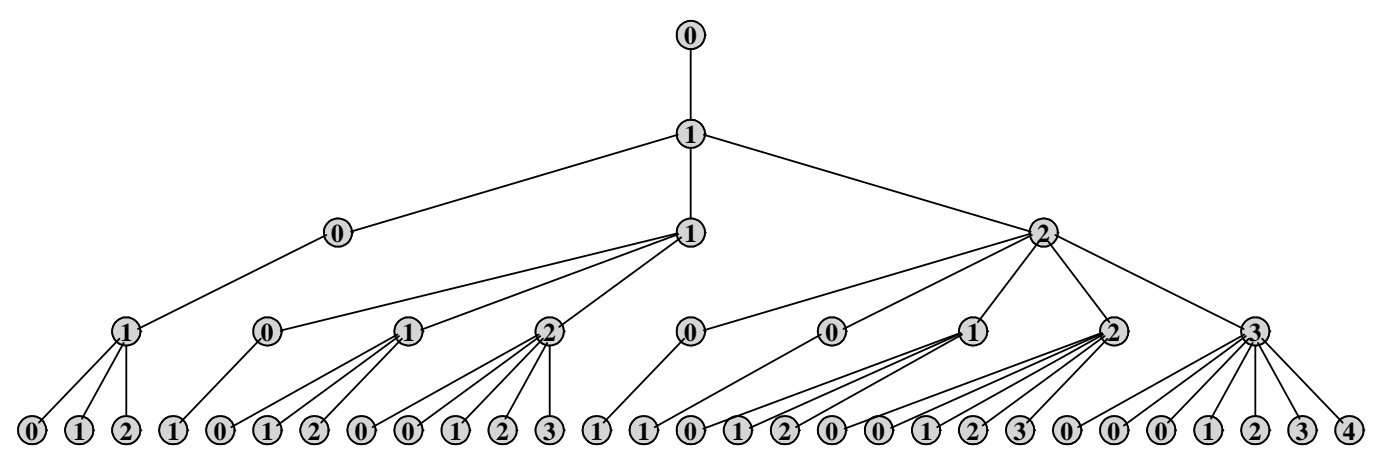

Fig. 4: The partial generating tree for the specification (11).

$$
=t+6 t^{2}+32 t^{3}+156 t^{4}+724 t^{5}+O\left(t^{6}\right)
$$

and the first few terms of this series development can be easily checked with Figure 4. Another example is given by the following quite general rule

$$
\left\{\begin{array}{ll}
\text { root : } & (0) \\
\text { rule: } & (k)
\end{array} \rightarrow(0)^{z_{k}}(k+1)\right.
$$

which corresponds to $A(t)=1$ and to a generic $Z(t)=\sum_{k \geq 0} z_{k} t^{k}$. The generating function $P(t)$ can be found as a function of $Z(t)$; in fact, in this case, formulas (4) and (5) give $h(t)=1$ and $d(t)=1 /(1-t Z(t))$, and we find:

$$
P(t)=\frac{t^{3}(1-t)^{2} Z^{\prime \prime}(t)+2 t^{2}(1-t)^{2} Z^{\prime}(t)-2 t^{2} Z(t)+2 t}{2(1-t)^{3}(1-t Z(t))^{2}} .
$$

Table 2 give some values of $P(t)$ for different $z_{k}$.

\begin{tabular}{|c|c|c|}
\hline$z_{k}$ & $Z(t)$ & $P(t)$ \\
\hline$p k$ & $\frac{p t}{(1-t)^{2}}$ & $\frac{\left(t^{2} p+p t+1-2 t+t^{2}\right) t}{\left(1-2 t+t^{2}-t^{2} p\right)^{2}(1-t)}=t+(3+p) t^{2}+(8 p+6) t^{3}+\left(30 p+2 p^{2}+10\right) t^{4}+O\left(t^{5}\right)$ \\
\hline$\frac{1}{k}\left(\begin{array}{c}2 k \\
k\end{array}\right)$ & $\frac{1-\sqrt{1-4 t}}{2 t}$ & $\frac{t\left((1-4 t)^{3 / 2}+1-6 t+12 t^{2}+2 t^{3}\right)}{2(1-4 t)^{3 / 2}(1+\sqrt{1-4 t})^{2}(1-t)^{3}}=t+5 t^{2}+20 t^{3}+83 t^{4}+366 t^{5}+O\left(t^{6}\right)$ \\
\hline$F_{k}$ & $\frac{t}{1-t-t^{2}}$ & $\frac{\left(2 t+3 t^{2}-11 t^{3}+6 t^{4}+3 t^{6}-1\right) t}{(1-t)^{3}(t+1)^{2}(2 t-1)^{2}\left(-1+t+t^{2}\right)}=t+4 t^{2}+11 t^{3}+32 t^{4}+83 t^{5}+O\left(t^{6}\right)$ \\
\hline
\end{tabular}

Tab. 2: Functions $P(t)$ corresponding to different $z_{k}$ in rule (12); $F_{k}$ denotes the $k$-th Fibonacci number.

Other examples concerning paths with an infinite set of jumps can be found in [Mer02]. 


\section{References}

$\left[\mathrm{BBMD}^{+}\right.$02] C. Banderier, M. Bousquet-Mélou, A. Denise, P. Flajolet, D. Gardy, and D. GouyouBeauchamps. Generating functions of generating trees. Discrete Mathematics, 246:29-55, 2002.

[BF02] C. Banderier and P. Flajolet. Basic analytic combinatorics of directed lattice paths. Theoretical Computer Science, 281(1-2):37-80, 2002.

[BK01] J. Bandlow and K. Killpatrick. An Area-to-Inv Bijection Between Dyck Paths and 312avoiding Permutations. The Electronic Jornal of Combinatorics, 8, 2001.

[BLPP99] E. Barcucci, A. Del Lungo, E. Pergola, and R. Pinzani. Eco: a methodology for the Enumeration of Combinatorial Objects. Journal of Difference Equations and Applications, 5:435-490, 1999.

[BM02] C. Banderier and D. Merlini. Lattice paths with an infinite set of jumps. Proceedings of the 14-th International Conference on Formal Power Series and Algebraic Combinatorics, Melbourne, Australia, 2002.

[CGHK78] F. R. K. Chung, R. L. Graham, V. E. Hoggat, and M. Kleiman. The number of Baxter permutations. Journal of Combinatorial Theory, Series A, 24:382-394, 1978.

[DF93] M. P. Delest and J. M. Fédou. Enumeration of skew Ferrers diagrams. Discrete Mathematics, 112:65-79, 1993.

[GJ83] I. P. Goulden and D. M. Jackson. Combinatorial Enumeration. John Wiley \& S., 1983.

[Knu73] D. E. Knuth. The art of computer programming. Vol. 1-3. Addison-Wesley, 1973.

[Mer02] D. Merlini. The internal path length of proper generating trees. Submitted, 2002.

[MRSV97] D. Merlini, D. G. Rogers, R. Sprugnoli, and M. C. Verri. On some alternative characterizations of Riordan arrays. Canadian Journal of Mathematics, 49(2):301-320, 1997.

[MRSV99] D. Merlini, D. G. Rogers, R. Sprugnoli, and M. C. Verri. Underdiagonal lattice paths with unrestricted steps. Discrete Applied Mathematics, 91:197-213, 1999.

[MSV94] D. Merlini, R. Sprugnoli, and M. C. Verri. Algebraic and combinatorial properties of simple, coloured walks. In Proceedings of CAAP'94, volume 787 of Lecture Notes in Computer Science, pages 218-233, 1994.

[MSV96] D. Merlini, R. Sprugnoli, and M. C. Verri. The area determined by underdiagonal lattice paths. In Proceedings of CAAP'96, volume 787 of Lecture Notes in Computer Science, pages 59-71, 1996.

[MV00] D. Merlini and M. C. Verri. Generating trees and proper Riordan Arrays. Discrete Mathematics, 218:167-183, 2000. 
[Rog78] D. G. Rogers. Pascal triangles, Catalan numbers and renewal arrays. Discrete Mathematics, 22:301-310, 1978.

[SGWW91] L. W. Shapiro, S. Getu, W.-J. Woan, and L. Woodson. The Riordan group. Discrete Applied Mathematics, 34:229-239, 1991.

[Spr94] R. Sprugnoli. Riordan arrays and combinatorial sums. Discrete Mathematics, 132:267-290, 1994.

[Su198] R. A. Sulanke. Bijective recurrences concerning Schröder paths. Electronic Journal of Combinatorics, 5(1), 1998.

[Sul00] B. Sulanke. Moments of generalized Motzkin paths. Journal of Integer Sequences, 3, 2000.

[Wes95] J. West. Generating trees and the Catalan and Schröder numbers. Discrete Mathematics, 146:247-262, 1995.

[Wes96] J. West. Generating trees and forbidden subsequences. Discrete Mathematics, 157:363374, 1996. 